CRYSTALLOGRAPHIC COMMUNICATIONS

ISSN 2056-9890

Received 2 September 2015

Accepted 16 September 2015

Edited by G. Smith, Queensland University of Technology, Australia

Keywords: crystal structure; proline; amino acid; strontium coordination polymer; N/O$\mathrm{H}$. . Br hydrogen bonds

CCDC reference: 1424731

Supporting information: this article has supporting information at journals.iucr.org/e

\section{Crystal structure of poly $\left[\left[\mu_{2}\right.\right.$-diaqua-diaqua- $\mu_{2}-\mathrm{L}-$ proline- $\kappa^{2} O: O^{\prime}$-strontium] dibromide]}

\author{
Selladurai Sathiskumar, ${ }^{\mathrm{a}}$ Thangavelu Balakrishnan, ${ }^{\mathrm{a} *}$ Kandasamy Ramamurthi ${ }^{\mathrm{b}}$ and \\ Subbiah Thamotharan ${ }^{\mathrm{c} *}$
}

${ }^{a}$ Crystal Growth Laboratory, PG and Research Department of Physics, Periyar EVR College (Autonomous), Tiruchirappalli 620 023, India, ${ }^{\mathbf{b}}$ Crystal Growth and Thin Film Laboratory, Department of Physics and Nanotechnology, SRM University, Kattankulathur 603 203, India, and 'Biomolecular Crystallography Laboratory, Department of Bioinformatics, School of Chemical and Biotechnology, SASTRA University, Thanjavur 613 401, India. *Correspondence e-mail:

balacrystalgrowth@gmail.com, thamu@scbt.sastra.edu

In the title coordination polymer, $\left\{\left[\mathrm{Sr}\left(\mathrm{C}_{5} \mathrm{H}_{9} \mathrm{NO}_{2}\right)\left(\mathrm{H}_{2} \mathrm{O}\right)_{4}\right] \mathrm{Br}_{2}\right\}_{n}$, the proline molecule exists in a zwitterionic form with one of the ring $\mathrm{C}$ atoms disordered over two sites [site-occupancy factors $=0.57(6): 0.43(6)]$. The $\mathrm{Sr}^{\mathrm{II}}$ ion is ninecoordinated by six water $\mathrm{O}$ atoms, two monodentate and two $\mu_{2}$-bridging, and three carboxylate $\mathrm{O}$ atoms of the proline ligands, with two bridging [ $\mathrm{Sr}-\mathrm{O}$ range $=2.524(4)-2.800(5) \AA]$. In the crystal, there is no direct interaction between the proline molecules. However, the proline and water molecules associate with the bromide counter-anions through a number of intermolecular $\mathrm{O}-\mathrm{H} \cdots \mathrm{Br}$ and $\mathrm{N}-\mathrm{H} \cdots \mathrm{Br}$ hydrogen-bonding interactions, giving a threedimensional supramolecular structure.

\section{Chemical context}

The study of coordination polymers has been an area of rapid development in recent years due to their interesting structures and their wide range of applications as functional materials (Lyhs et al., 2012). Reports of the crystal structures of alkaline earth metal ions combined with anions of amino acids are very limited. As part of our ongoing investigations of the crystal and molecular structures of a series of metal complexes generated from amino acids (Revathi et al., 2015; Sathiskumar et al., 2015a,b; Balakrishnan et al., 2013), we report here the crystal structure of a polymeric strontium-proline complex, $\left\{\left[\mathrm{Sr}\left(\mathrm{C}_{5} \mathrm{H}_{9} \mathrm{NO}_{2}\right)\left(\mathrm{H}_{2} \mathrm{O}\right)_{4}\right]^{2+} 2\left(\mathrm{Br}^{-}\right)\right\}_{n},(\mathrm{I})$.

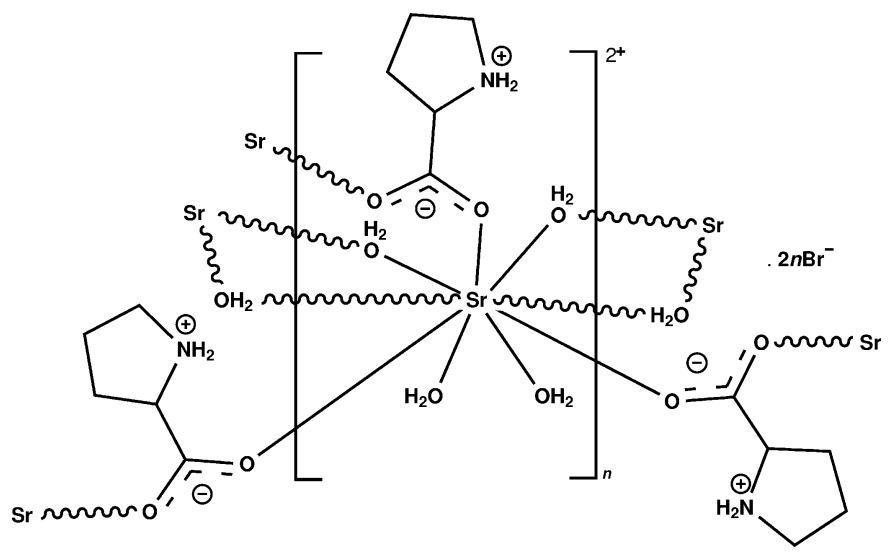

\section{Structural commentary}

The asymmetric unit of the title complex (I) contains one $\mathrm{Sr}^{2+}$ ion, one bridging proline ligand and four water molecules, two of which are monodentate and two bridging, and two bromide 


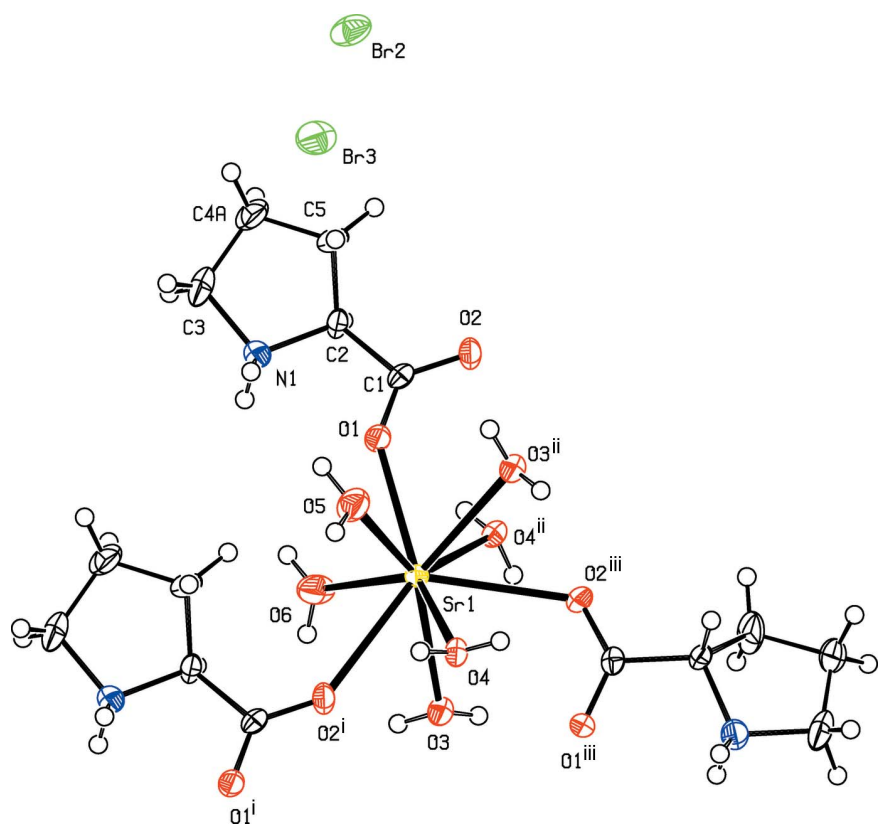

Figure 1

The coordination sphere of $\mathrm{Sr}^{2+}$ in the crystal structure of (I). Only the major components of the disordered proline ligands are shown. Displacement ellipsoids are drawn at the $50 \%$ probability level. For symmetry codes, see Table 1 .

counter-anions (Fig. 1). In (I), the bond lengths involving the carboxylate atoms and the protonation of the amino group suggest that the proline molecule exists in a zwitterionic form. The $\mathrm{Sr}^{\mathrm{II}}$ ion is nine-coordinated by six water oxygen atoms

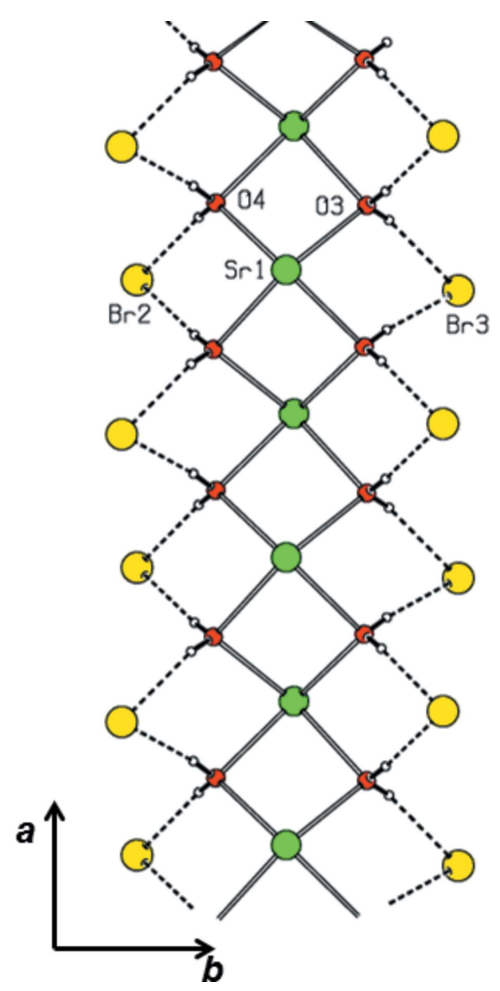

Figure 2

The Sr-water coordination polymeric chain substructure of (I), with peripheral water $\mathrm{O}-\mathrm{H} \cdots \mathrm{Br}$ hydrogen bonds shown as dashed lines.
Table 1

Selected bond lengths $(\AA)$.

\begin{tabular}{llll}
\hline $\mathrm{Sr} 1-\mathrm{O} 1$ & $2.524(4)$ & $\mathrm{Sr} 1-\mathrm{O} 2^{\mathrm{i}}$ & $2.728(4)$ \\
$\mathrm{Sr} 1-\mathrm{O} 3$ & $2.625(6)$ & $\mathrm{Sr} 1-\mathrm{O} 3^{\mathrm{ii}}$ & $2.707(6)$ \\
$\mathrm{Sr} 1-\mathrm{O} 4$ & $2.630(6)$ & $\mathrm{Sr} 1-\mathrm{O} 4^{\mathrm{ii}}$ & $2.651(5)$ \\
$\mathrm{Sr} 1-\mathrm{O} 5$ & $2.593(5)$ & $\mathrm{Sr} 1-\mathrm{O} 2^{\mathrm{iii}}$ & $2.800(5)$ \\
$\mathrm{Sr} 1-\mathrm{O} 6$ & $2.582(6)$ & & \\
\hline
\end{tabular}

Symmetry codes: (i) $x+1, y, z$; (ii) $x-\frac{1}{2},-y+\frac{1}{2},-z+1$; (iii) $x+\frac{1}{2},-y+\frac{1}{2},-z+1$.

Table 2

Hydrogen-bond geometry $\left(\AA{ }^{\circ}\right)$.

\begin{tabular}{|c|c|c|c|c|}
\hline$D-\mathrm{H} \cdots A$ & $D-\mathrm{H}$ & $\mathrm{H} \cdots A$ & $D \cdots A$ & $D-\mathrm{H} \cdots A$ \\
\hline $\mathrm{N} 1-\mathrm{H} 1 A \cdots \mathrm{Br} 2^{\mathrm{i}}$ & $0.90(6)$ & $2.52(5)$ & $3.374(7)$ & $159(6)$ \\
\hline $\mathrm{N} 1-\mathrm{H} 1 B \cdots \mathrm{Br} 3^{\mathrm{i}}$ & $0.90(7)$ & $2.40(7)$ & $3.240(7)$ & $156(8)$ \\
\hline $\mathrm{O} 3-\mathrm{H} 3 C \cdots \mathrm{Br} 3^{\mathrm{iv}}$ & $0.84(7)$ & $2.63(7)$ & $3.440(6)$ & $163(7)$ \\
\hline $\mathrm{O} 3-\mathrm{H} 3 D \cdots \mathrm{Br}^{\mathrm{v}}$ & $0.84(7)$ & $2.54(7)$ & $3.376(6)$ & $172(5)$ \\
\hline $\mathrm{O} 4-\mathrm{H} 4 E \cdots \mathrm{Br} 2^{\mathrm{vi}}$ & $0.85(6)$ & $2.47(7)$ & $3.281(6)$ & $162(7)$ \\
\hline $\mathrm{O} 4-\mathrm{H} 4 F \cdots \mathrm{Br} 3^{\mathrm{vii}}$ & $0.83(6)$ & $2.52(6)$ & $3.347(6)$ & $174(6)$ \\
\hline $\mathrm{O} 5-\mathrm{H} 5 \mathrm{C} \cdots \mathrm{Br}^{\mathrm{i}}$ & $0.86(5)$ & $2.54(5)$ & $3.369(6)$ & $164(6)$ \\
\hline $\mathrm{O} 5-\mathrm{H} 5 D \cdots \mathrm{Br} 3^{\mathrm{vii}}$ & $0.84(6)$ & $2.48(6)$ & $3.304(6)$ & $166(6)$ \\
\hline $\mathrm{O} 6-\mathrm{H} 6 C \cdots \mathrm{Br} 2^{\mathrm{v}}$ & $0.83(6)$ & $2.58(6)$ & $3.393(6)$ & $167(5)$ \\
\hline $\mathrm{O} 6-\mathrm{H} 6 D \cdots \mathrm{Br} 3^{\mathrm{i}}$ & $0.85(7)$ & $2.56(6)$ & $3.378(6)$ & $162(7)$ \\
\hline
\end{tabular}

Symmetry codes: (i) $x+1, y, z$; (iv) $-x+\frac{3}{2},-y+1, z+\frac{1}{2}$; (v) $-x+2, y+\frac{1}{2},-z+\frac{1}{2}$; (vi) $-x+\frac{3}{2},-y, z+\frac{1}{2}$; (vii) $-x+2, y-\frac{1}{2},-z+\frac{1}{2}$.

$[\mathrm{Sr}-\mathrm{O}=2.582(6)-2.707(5) \AA]$ and three carboxylate oxygen atoms of zwitterionic proline ligands $[\mathrm{Sr}-\mathrm{O}=2.524$ (4)2.800 (4) $\AA$; Table 1]. In the strontium-glycine complex, the $\mathrm{Sr}-\mathrm{O}$ (water) and $\mathrm{Sr}-\mathrm{O}$ (carboxylate) distances ranges are 2.526 (4)-2.661 (2) and $2.605(2)-2.703$ (2) ̊, respectively (Revathi et al., 2015). In (I), one of the carbon atoms (C4) of the pyrrolidine ring is disordered over two sites. In the major component of the pyrrolidine ring, there is a twist conformation on the $\mathrm{C} 2-\mathrm{C} 5$ bond with a pseudo-rotation angle $\Delta=$ $40.1(14)^{\circ}$ and a maximum torsion angle $\varphi_{\mathrm{m}}=43.8(10)^{\circ}$ for the atom sequence N1-C2-C5-C4A-C3 (Rao et al., 1981). In the minor component, the pyrrolidine ring exhibits an envelope conformation on $\mathrm{N} 1$ with a pseudo-rotation angle $\Delta=$ $341.5(19)^{\circ}$ and a maximum torsion angle $\varphi_{\mathrm{m}}=36.0(9)^{\circ}$ for the atom sequence $\mathrm{N} 1-\mathrm{C} 2-\mathrm{C} 5-\mathrm{C} 4 B-\mathrm{C} 3$ (Rao et al., 1981). As shown in Fig. 2, the title complex forms a coordination polymeric chain that lies parallel to the $a$ axis. Adjacent $\mathrm{Sr}^{\mathrm{II}}$ ions are separated by 3.9387 (7) Å within a chain.

\section{Supramolecular features}

The crystal structure of (I), is stabilized by intermolecular $\mathrm{N}-$ $\mathrm{H} \cdots \mathrm{Br}$ and $\mathrm{O}-\mathrm{H} \cdots \mathrm{Br}$ hydrogen bonds (Table 2). One of the characteristic features observed in amino acid complexes is the head-to-tail sequence in which amino acids are self-associated through their amino and carboxylate groups (Sharma et al., 2006; Selvaraj et al., 2007; Balakrishnan et al., 2013; Revathi et al., 2015). In the crystal structure of the L-proline lithium bromide monohydrate complex, there is a head-to-tail sequence observed (Sathiskumar et al., 2015a). In contrast, there is no direct hydrogen-bonding interaction between the proline molecules in (I). 


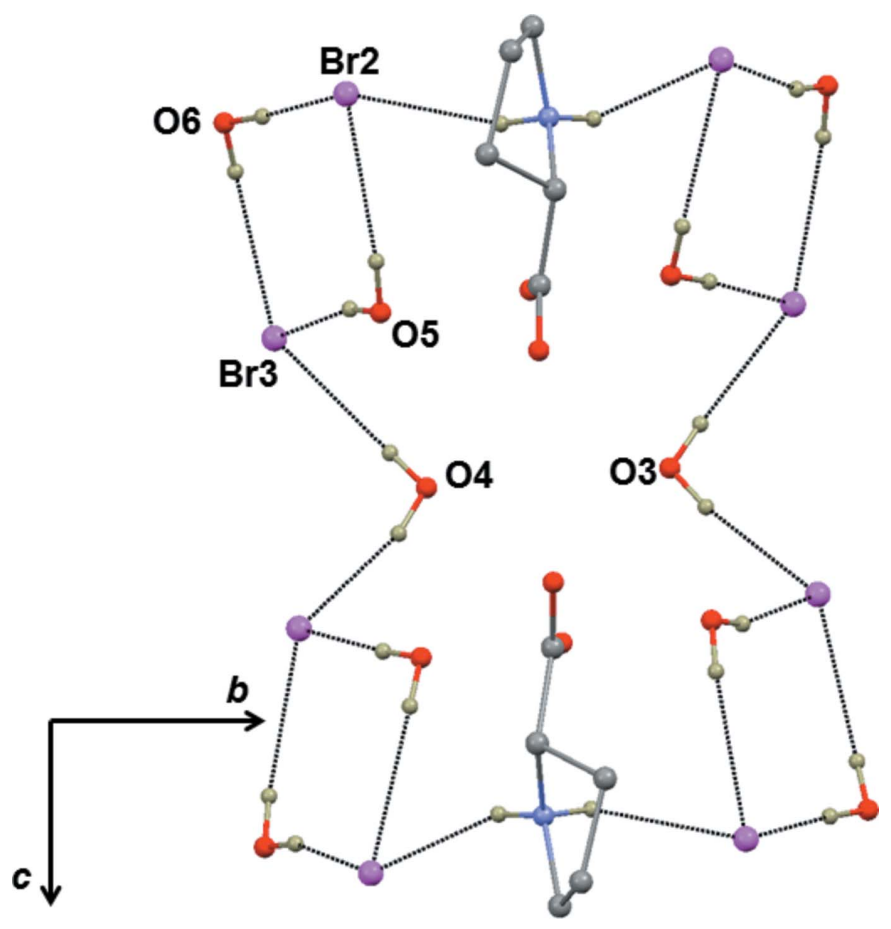

Figure 3

The butterfly-like supramolecular arrangements generated by intermolecular $\mathrm{N}-\mathrm{H} \cdots \mathrm{Br}$ and $\mathrm{O}-\mathrm{H} \cdots \mathrm{Br}$ hydrogen bonds. Only atoms involved in hydrogen-bonding interactions are labelled.



Figure 4

The crystal packing of (I) viewed along the $a$ axis, with hydrogen bonds shown as dashed lines. C-bound $\mathrm{H}$ atoms have been omitted for clarity.
Table 3

Experimental details.

\begin{tabular}{|c|c|}
\hline \multicolumn{2}{|l|}{ Crystal data } \\
\hline Chemical formula & {$\left[\mathrm{Sr}\left(\mathrm{C}_{5} \mathrm{H}_{9} \mathrm{NO}_{2}\right)\left(\mathrm{H}_{2} \mathrm{O}\right)_{4}\right] \mathrm{Br}_{2}$} \\
\hline$M_{\mathrm{r}}$ & 434.63 \\
\hline Crystal system, space group & Orthorhombic, $P 2_{1} 2_{1} 2_{1}$ \\
\hline Temperature $(\mathrm{K})$ & 296 \\
\hline$a, b, c(\AA)$ & $\begin{array}{l}6.7079(4), 12.9125(9) \\
15.4499(11)\end{array}$ \\
\hline$V\left(\AA^{3}\right)$ & $1338.20(16)$ \\
\hline$Z$ & 4 \\
\hline Radiation type & Мо $K \alpha$ \\
\hline$\mu\left(\mathrm{mm}^{-1}\right)$ & 10.01 \\
\hline Crystal size $(\mathrm{mm})$ & $0.15 \times 0.10 \times 0.10$ \\
\hline \multicolumn{2}{|l|}{ Data collection } \\
\hline Diffractometer & Bruker Kappa APEXII CCD \\
\hline Absorption correction & $\begin{array}{l}\text { Multi-scan ( } S A B A B S ; \text { Bruker, } \\
\text { 2004) }\end{array}$ \\
\hline$T_{\min }, T_{\max }$ & $0.26,0.44$ \\
\hline $\begin{array}{l}\text { No. of measured, independent and } \\
\text { observed }[I>2 \sigma(I)] \text { reflections }\end{array}$ & $14183,2345,2081$ \\
\hline$R_{\text {int }}$ & 0.068 \\
\hline$(\sin \theta / \lambda)_{\max }\left(\AA^{-1}\right)$ & 0.594 \\
\hline \multicolumn{2}{|l|}{ Refinement } \\
\hline$R\left[F^{2}>2 \sigma\left(F^{2}\right)\right], w R\left(F^{2}\right), S$ & $0.032,0.063,1.07$ \\
\hline No. of reflections & 2345 \\
\hline No. of parameters & 186 \\
\hline No. of restraints & 26 \\
\hline $\mathrm{H}$-atom treatment & $\begin{array}{l}\mathrm{H} \text { atoms treated by a mixture of } \\
\text { independent and constrained } \\
\text { refinement }\end{array}$ \\
\hline$\Delta \rho_{\max }, \Delta \rho_{\min }\left(\mathrm{e} \AA^{-3}\right)$ & $0.60,-0.86$ \\
\hline Absolute structure & $\begin{array}{l}\text { Flack } x \text { determined using } 788 \\
\quad \text { quotients }\left[\left(I^{+}\right)-\left(I^{-}\right)\right] /\left[\left(I^{+}\right)+\left(I^{-}\right)\right] \\
\quad \text { (Parsons } \text { et al., 2013) }\end{array}$ \\
\hline Absolute structure parameter & $0.008(8)$ \\
\hline
\end{tabular}

Computer programs: APEX2, SAINT and XPREP (Bruker, 2004), SIR92 (Altomare et al., 1993), SHELXL2014 (Sheldrick, 2015), PLATON (Spek, 2009) and Mercury (Macrae et al., 2008).

As shown in Fig. 3, two water molecules and two bromide anions along with $\mathrm{Sr}^{2+}$ ions generate a hydrogen-bonded sheet which lies parallel to the $a$ axis. Within this sheet, two $\mathrm{Sr}^{2+}$ ions and two water oxygens form a cyclic motif. Water molecules (O3 and O4) interconnect the bromide anions, forming a chain. In (I), two molecules (O5 and O6) act as donors for intermolecular $\mathrm{O}-\mathrm{H} \cdots \mathrm{Br}$ hydrogen bonds. These hydrogen bonds generate a cyclic dibromide motif similar to that observed in a related structure (Revathi et al., 2015). Adjacent dibromide motifs in (I), which run parallel to the $b$ axis, are interconnected by proline ligands through intermolecular $\mathrm{N}-$ $\mathrm{H} \cdots \mathrm{Br}$ hydrogen bonds on both sides (Fig. 3). Adjacent supramolecular arrangements of cyclic dibromide .. proline . . cyclic dibromide motifs are interlinked further by water molecules (O3 and $\mathrm{O} 4)$ through $\mathrm{O}-\mathrm{H} \cdots \mathrm{Br}$ hydrogen bonds. This entire arrangement forms a butterfly-like structure. The overall hydrogen-bonded supramolecular structure (Fig. 4) is three-dimensional.

\section{Synthesis and crystallization}

Single crystals of the title complex were obtained by slow evaporation from an aqueous solution of L-proline and 
strontium bromide hexahydrate in a 1:1 stoichiometric molar ratio at $306 \mathrm{~K}$. The prepared solution was stirred well and filtered. The resultant filtered solution was left undisturbed to allow evaporation. After 15 days, colourless prismatic crystals were harvested.

\section{Refinement}

Crystal data, data collection and structure refinement details are summarized in Table 3. One of the carbon (C4) atoms of the pyrrolidine ring appears to be disordered over two sites. These positions were defined for this atom and constrained refinement of the site-occupation factors led to a value of 0.57 (6) for the major component. The positions of amino and water $\mathrm{H}$ atoms were located from difference Fourier maps. Further, the $\mathrm{O}-\mathrm{H}$ distances in the water molecules were restrained to 0.85 (2) $\AA$. The $\mathrm{N}-\mathrm{H}$ distances of amino group were also restrained, to 0.89 (2) $\AA$. The remaining hydrogen atoms were placed in geometrically idealized positions $(\mathrm{C}-\mathrm{H}$ $=0.97 \AA$ with $U_{\text {iso }}(\mathrm{H})=1.2 U_{\text {eq }}(\mathrm{C})$ and were constrained to ride on their parent atom. The Flack absolute structure parameter was determined to be 0.008 (8) (788 Friedel pairs; Parsons et al., 2013), indicating an $S$ configuration for C2, consistent with that for the parent L-proline (Kayushina \& Vainshtein, 1965).

\section{Acknowledgements}

TB and SS would like to acknowledge the University Grants Commission (UGC), India for providing financial support
[Project No. 41-956/2012(SR)]. ST is very grateful to the management of SASTRA University for infrastructural and financial support (Professor TRR grant).

\section{References}

Altomare, A., Cascarano, G., Giacovazzo, C. \& Guagliardi, A. (1993). J. Appl. Cryst. 26, 343-350.

Balakrishnan, T., Ramamurthi, K., Jeyakanthan, J. \& Thamotharan, S. (2013). Acta Cryst. E69, m60-m61.

Bruker (2004). APEX2, SAINT, XPREP and SADABS. Bruker AXS Inc., Madison, Wisconsin, USA.

Kayushina, R. L. \& Vainshtein, B. K. (1965). Kristallografiya, 10, $833-$ 844.

Lyhs, B., Bläser, D., Wölper, C., Haack, R., Jansen, G. \& Schulz, S. (2012). Eur. J. Inorg. Chem. pp. 4350-4355.

Macrae, C. F., Bruno, I. J., Chisholm, J. A., Edgington, P. R., McCabe, P., Pidcock, E., Rodriguez-Monge, L., Taylor, R., van de Streek, J. \& Wood, P. A. (2008). J. Appl. Cryst. 41, 466-470.

Parsons, S., Flack, H. D. \& Wagner, T. (2013). Acta Cryst. B69, 249259.

Rao, S. T., Westhof, E. \& Sundaralingam, M. (1981). Acta Cryst. A37, 421-425.

Revathi, P., Balakrishnan, T., Ramamurthi, K. \& Thamotharan, S. (2015). Acta Cryst. E71, 875-878.

Sathiskumar, S., Balakrishnan, T., Ramamurthi, K. \& Thamotharan, S. (2015a). Spectrochim. Acta Part A, 138, 187-194.

Sathiskumar, S., Balakrishnan, T., Ramamurthi, K. \& Thamotharan, S. (2015b). Acta Cryst. E71, 217-219.

Selvaraj, M., Thamotharan, S., Roy, S. \& Vijayan, M. (2007). Acta Cryst. B63, 459-468.

Sharma, A., Thamotharan, S., Roy, S. \& Vijayan, M. (2006). Acta Cryst. C62, o148-o152.

Sheldrick, G. M. (2015). Acta Cryst. C71, 3-8.

Spek, A. L. (2009). Acta Cryst. D65, 148-155. 


\section{supporting information}

Acta Cryst. (2015). E71, 1199-1202 [doi:10.1107/S2056989015017302]

\section{Crystal structure of poly[[$\mu_{2}$-diaqua-diaqua- $\mu_{2}$-L-proline- $\kappa^{2} O: O^{\prime}$-strontium $]$ dibromide]}

\section{Selladurai Sathiskumar, Thangavelu Balakrishnan, Kandasamy Ramamurthi and Subbiah \\ Thamotharan}

\section{Computing details}

Data collection: APEX2 (Bruker, 2004); cell refinement: APEX2 and SAINT (Bruker, 2004); data reduction: SAINT and XPREP (Bruker, 2004); program(s) used to solve structure: SIR92 (Altomare et al., 1993); program(s) used to refine structure: SHELXL2014 (Sheldrick, 2015); molecular graphics: PLATON (Spek, 2009) and Mercury (Macrae et al., 2008); software used to prepare material for publication: SHELXL2014 (Sheldrick, 2015).

Poly $\left[\left[\mu_{2}\right.\right.$-diaqua-diaqua- $\mu_{2}-L-$ proline- $\kappa^{2} O: O^{\prime}-\backslash$ strontium $]$ dibromide $]$

Crystal data

$\left[\mathrm{Sr}\left(\mathrm{C}_{5} \mathrm{H}_{9} \mathrm{NO}_{2}\right)\left(\mathrm{H}_{2} \mathrm{O}\right)_{4}\right] \mathrm{Br}_{2}$

$M_{r}=434.63$

Orthorhombic, $P 22_{1} 2_{1} 2_{1}$

$a=6.7079(4) \AA$

$b=12.9125(9) \AA$

$c=15.4499(11) \AA$

$V=1338.20(16) \AA^{3}$

$Z=4$

$F(000)=840$

Data collection

Bruker Kappa APEXII CCD diffractometer

Radiation source: Sealed tube $\omega$ nd $\varphi$ scan

Absorption correction: multi-scan

(SABABS; Bruker, 2004)

$T_{\min }=0.26, T_{\max }=0.44$

14183 measured reflections

Refinement

Refinement on $F^{2}$

Least-squares matrix: full

$R\left[F^{2}>2 \sigma\left(F^{2}\right)\right]=0.032$

$w R\left(F^{2}\right)=0.063$

$S=1.07$

2345 reflections

186 parameters

26 restraints

Hydrogen site location: mixed
$D_{\mathrm{x}}=2.157 \mathrm{Mg} \mathrm{m}^{-3}$

Mo $K \alpha$ radiation, $\lambda=0.71073 \AA$

Cell parameters from 7063 reflections

$\theta=2.6-28.5^{\circ}$

$\mu=10.01 \mathrm{~mm}^{-1}$

$T=296 \mathrm{~K}$

Block, brown

$0.15 \times 0.10 \times 0.10 \mathrm{~mm}$

2345 independent reflections

2081 reflections with $I>2 \sigma(I)$

$R_{\text {int }}=0.068$

$\theta_{\text {max }}=25.0^{\circ}, \theta_{\min }=2.6^{\circ}$

$h=-7 \rightarrow 7$

$k=-15 \rightarrow 15$

$l=-18 \rightarrow 18$

$\mathrm{H}$ atoms treated by a mixture of independent and constrained refinement

$w=1 /\left[\sigma^{2}\left(F_{\mathrm{o}}^{2}\right)+(0.0267 P)^{2}\right]$

where $P=\left(F_{\mathrm{o}}^{2}+2 F_{\mathrm{c}}^{2}\right) / 3$

$(\Delta / \sigma)_{\max }<0.001$

$\Delta \rho_{\max }=0.60 \mathrm{e} \AA^{-3}$

$\Delta \rho_{\min }=-0.86$ e $\AA^{-3}$ 
Absolute structure: Flack $x$ determined using

788 quotients $\left[\left(I^{+}\right)-(I)\right] /\left[\left(I^{+}\right)+(I)\right]$ (Parsons et al., 2013)

Absolute structure parameter: 0.008 (8)

Special details

Geometry. Bond distances, angles etc. have been calculated using the rounded fractional coordinates. All su's are estimated from the variances of the (full) variance-covariance matrix. The cell esds are taken into account in the estimation of distances, angles and torsion angles

Fractional atomic coordinates and isotropic or equivalent isotropic displacement parameters $\left(\hat{A}^{2}\right)$

\begin{tabular}{|c|c|c|c|c|c|}
\hline & $x$ & $y$ & $z$ & $U_{\text {iso }} * / U_{\text {eq }}$ & Occ. $(<1)$ \\
\hline Sr1 & $1.34882(8)$ & $0.24302(5)$ & $0.43342(4)$ & $0.0174(2)$ & \\
\hline O1 & $1.0492(6)$ & $0.2322(4)$ & $0.3350(3)$ & $0.0257(16)$ & \\
\hline $\mathrm{O} 2$ & $0.7439(6)$ & $0.2420(4)$ & $0.3916(3)$ & $0.0243(16)$ & \\
\hline $\mathrm{O} 3$ & $1.5725(8)$ & $0.3885(5)$ & $0.5016(4)$ & $0.0243(19)$ & \\
\hline $\mathrm{O} 4$ & $1.5819(8)$ & $0.1162(4)$ & $0.5200(4)$ & $0.0213(17)$ & \\
\hline $\mathrm{O} 5$ & $1.3443(9)$ & $0.0648(4)$ & $0.3561(4)$ & $0.035(2)$ & \\
\hline O6 & $1.3860(9)$ & $0.3899(5)$ & $0.3212(4)$ & $0.038(2)$ & \\
\hline N1 & $0.9404(8)$ & $0.2529(6)$ & $0.1734(4)$ & $0.026(2)$ & \\
\hline $\mathrm{C} 1$ & $0.8660(9)$ & $0.2426(5)$ & $0.3307(4)$ & $0.018(2)$ & \\
\hline $\mathrm{C} 2$ & $0.7837(9)$ & $0.2600(6)$ & $0.2411(4)$ & $0.021(2)$ & \\
\hline $\mathrm{C} 3$ & $0.8370(12)$ & $0.2347(7)$ & $0.0890(5)$ & $0.042(3)$ & \\
\hline $\mathrm{C} 4 \mathrm{~A}$ & $0.623(2)$ & $0.211(3)$ & $0.1117(11)$ & $0.034(7)$ & $0.57(6)$ \\
\hline $\mathrm{C} 5$ & $0.6277(12)$ & $0.1840(7)$ & $0.2082(5)$ & $0.042(3)$ & \\
\hline C4B & $0.660(5)$ & 0.167 (3) & $0.1117(13)$ & $0.035(8)$ & $0.43(6)$ \\
\hline $\mathrm{Br} 2$ & $0.18627(12)$ & $0.02641(6)$ & $0.15165(6)$ & $0.0389(3)$ & \\
\hline $\mathrm{Br} 3$ & $0.22307(13)$ & $0.44596(7)$ & $0.11973(6)$ & $0.0466(3)$ & \\
\hline H1A & $1.024(9)$ & 0.199 (4) & $0.180(5)$ & $0.02(2)^{*}$ & \\
\hline H1B & $1.033(10)$ & $0.303(5)$ & $0.175(6)$ & $0.05(3)^{*}$ & \\
\hline $\mathrm{H} 3 \mathrm{C}$ & $1.522(13)$ & $0.429(5)$ & $0.538(4)$ & $0.06(3)^{*}$ & \\
\hline H3D & $1.622(13)$ & $0.422(5)$ & $0.460(4)$ & $0.07(4)^{*}$ & \\
\hline $\mathrm{H} 4 \mathrm{E}$ & $1.532(12)$ & $0.085(5)$ & $0.563(3)$ & $0.05(3)^{*}$ & \\
\hline $\mathrm{H} 4 \mathrm{~F}$ & $1.640(10)$ & $0.075(4)$ & $0.487(4)$ & $0.04(3)^{*}$ & \\
\hline $\mathrm{H} 5 \mathrm{C}$ & $1.281(10)$ & $0.060(6)$ & $0.308(3)$ & $0.06(3)^{*}$ & \\
\hline H5D & $1.450(7)$ & $0.030(6)$ & $0.353(5)$ & $0.06(3)^{*}$ & \\
\hline $\mathrm{H} 6 \mathrm{C}$ & $1.478(8)$ & $0.432(5)$ & $0.327(5)$ & $0.03(3)^{*}$ & \\
\hline H6D & $1.319(11)$ & $0.402(7)$ & $0.276(4)$ & $0.09(4)^{*}$ & \\
\hline H31 & 0.89720 & 0.17700 & 0.05850 & $0.0500 *$ & $0.57(6)$ \\
\hline H32 & 0.84480 & 0.29580 & 0.05270 & $0.0500^{*}$ & $0.57(6)$ \\
\hline H41 & 0.57440 & 0.15290 & 0.07800 & $0.0410^{*}$ & $0.57(6)$ \\
\hline $\mathrm{H} 42$ & 0.53890 & 0.27050 & 0.10120 & $0.0410^{*}$ & $0.57(6)$ \\
\hline H51 & 0.49920 & 0.19560 & 0.23530 & $0.0500^{*}$ & $0.57(6)$ \\
\hline H52 & 0.66830 & 0.11280 & 0.21770 & $0.0500^{*}$ & $0.57(6)$ \\
\hline $\mathrm{H} 2$ & 0.72630 & 0.32970 & 0.23900 & $0.0250^{*}$ & \\
\hline H33 & 0.92450 & 0.19970 & 0.04850 & $0.0500^{*}$ & $0.43(6)$ \\
\hline H34 & 0.79360 & 0.29960 & 0.06370 & $0.0500^{*}$ & $0.43(6)$ \\
\hline H43 & 0.68850 & 0.09490 & 0.09960 & $0.0420^{*}$ & $0.43(6)$ \\
\hline
\end{tabular}


supporting information

\begin{tabular}{|c|c|c|c|c|c|}
\hline $\mathrm{H} 44$ & 0.54310 & 0.18790 & 0.07910 & $0.0420 *$ & $0.43(6)$ \\
\hline H53 & 0.49510 & 0.21140 & 0.21840 & $0.0500 *$ & $0.43(6)$ \\
\hline H54 & 0.63970 & 0.11870 & 0.23880 & $0.0500 *$ & $0.43(6)$ \\
\hline
\end{tabular}

Atomic displacement parameters $\left(\AA^{2}\right)$

\begin{tabular}{lllllll}
\hline & $U^{11}$ & $U^{22}$ & $U^{33}$ & $U^{12}$ & $U^{13}$ & $U^{23}$ \\
\hline Sr1 & $0.0142(3)$ & $0.0215(4)$ & $0.0165(3)$ & $0.0003(3)$ & $-0.0010(3)$ & $0.0006(3)$ \\
O1 & $0.017(2)$ & $0.041(3)$ & $0.019(3)$ & $0.006(2)$ & $-0.001(2)$ & $-0.003(3)$ \\
O2 & $0.024(2)$ & $0.033(3)$ & $0.016(3)$ & $0.004(3)$ & $0.004(2)$ & $0.002(3)$ \\
O3 & $0.025(3)$ & $0.029(3)$ & $0.019(4)$ & $0.000(2)$ & $0.000(3)$ & $-0.003(3)$ \\
O4 & $0.024(3)$ & $0.024(3)$ & $0.016(3)$ & $0.000(2)$ & $0.003(3)$ & $0.000(3)$ \\
O5 & $0.033(3)$ & $0.044(4)$ & $0.029(4)$ & $0.006(3)$ & $-0.002(3)$ & $-0.010(3)$ \\
O6 & $0.034(4)$ & $0.043(4)$ & $0.037(4)$ & $-0.012(3)$ & $-0.011(3)$ & $0.017(3)$ \\
N1 & $0.022(3)$ & $0.035(4)$ & $0.022(4)$ & $-0.005(4)$ & $0.000(3)$ & $0.004(4)$ \\
C1 & $0.025(4)$ & $0.011(3)$ & $0.017(4)$ & $-0.001(3)$ & $-0.003(3)$ & $-0.004(3)$ \\
C2 & $0.024(3)$ & $0.026(4)$ & $0.013(4)$ & $0.005(4)$ & $0.002(3)$ & $0.000(4)$ \\
C3 & $0.052(5)$ & $0.059(6)$ & $0.015(4)$ & $0.000(5)$ & $-0.001(4)$ & $-0.001(4)$ \\
C4A & $0.036(10)$ & $0.041(15)$ & $0.025(9)$ & $0.001(8)$ & $-0.007(7)$ & $-0.014(9)$ \\
C5 & $0.035(5)$ & $0.066(6)$ & $0.025(5)$ & $-0.025(4)$ & $-0.006(4)$ & $-0.004(5)$ \\
C4B & $0.033(13)$ & $0.042(17)$ & $0.030(11)$ & $-0.010(13)$ & $0.005(10)$ & $-0.023(11)$ \\
Br2 & $0.0357(5)$ & $0.0379(5)$ & $0.0432(5)$ & $0.0057(4)$ & $-0.0102(4)$ & $-0.0197(4)$ \\
Br3 & $0.0505(6)$ & $0.0484(6)$ & $0.0409(6)$ & $-0.0239(4)$ & $-0.0107(4)$ & $0.0197(5)$ \\
& & & & & &
\end{tabular}

Geometric parameters $\left(\AA,{ }^{\circ}\right)$

\begin{tabular}{llll}
\hline $\mathrm{Sr} 1-\mathrm{O} 1$ & $2.524(4)$ & $\mathrm{N} 1-\mathrm{H} 1 \mathrm{~A}$ & $0.90(6)$ \\
$\mathrm{Sr} 1-\mathrm{O} 3$ & $2.625(6)$ & $\mathrm{N} 1-\mathrm{H} 1 \mathrm{~B}$ & $0.90(7)$ \\
$\mathrm{Sr} 1-\mathrm{O} 4$ & $2.630(6)$ & $\mathrm{C} 1-\mathrm{C} 2$ & $1.507(9)$ \\
$\mathrm{Sr} 1-\mathrm{O} 5$ & $2.593(5)$ & $\mathrm{C} 2-\mathrm{C} 5$ & $1.522(11)$ \\
$\mathrm{Sr} 1-\mathrm{O} 6$ & $2.582(6)$ & $\mathrm{C} 3-\mathrm{C} 4 \mathrm{~A}$ & $1.509(17)$ \\
$\mathrm{Sr} 1-\mathrm{O} 2^{\mathrm{i}}$ & $2.728(4)$ & $\mathrm{C} 3-\mathrm{C} 4 \mathrm{~B}$ & $1.52(4)$ \\
$\mathrm{Sr} 1-\mathrm{O} 3^{\mathrm{ii}}$ & $2.707(6)$ & $\mathrm{C} 4 \mathrm{~A}-\mathrm{C} 5$ & $1.53(2)$ \\
$\mathrm{Sr} 1-\mathrm{O} 4$ & $\mathrm{C} 4 \mathrm{~B}-\mathrm{C} 5$ & $1.52(2)$ \\
$\mathrm{Sr} 1-\mathrm{O} 2^{\mathrm{iii}}$ & $2.651(5)$ & $\mathrm{C} 2-\mathrm{H} 2$ & 0.9800 \\
$\mathrm{O} 1-\mathrm{C} 1$ & $2.800(5)$ & $\mathrm{C} 3-\mathrm{H} 31$ & 0.9700 \\
$\mathrm{O} 2-\mathrm{C} 1$ & $1.238(7)$ & $\mathrm{C} 3-\mathrm{H} 32$ & 0.9700 \\
$\mathrm{O} 3-\mathrm{H} 3 \mathrm{C}$ & $1.248(8)$ & $\mathrm{C} 3-\mathrm{H} 33$ & 0.9700 \\
$\mathrm{O} 3-\mathrm{H} 3 \mathrm{D}$ & $0.84(7)$ & $\mathrm{C} 3-\mathrm{H} 34$ & 0.9700 \\
$\mathrm{O} 4-\mathrm{H} 4 \mathrm{E}$ & $0.84(7)$ & $\mathrm{C} 4 \mathrm{~A}-\mathrm{H} 41$ & 0.9700 \\
$\mathrm{O} 4-\mathrm{H} 4 \mathrm{~F} 42$ & 0.9700 \\
$\mathrm{O} 5-\mathrm{H} 5 \mathrm{D}$ & $0.85(6)$ & $\mathrm{C} 4 \mathrm{~B}-\mathrm{H} 43$ & 0.9700 \\
$\mathrm{O} 5-\mathrm{H} 5 \mathrm{C}$ & $0.83(6)$ & $\mathrm{C} 4 \mathrm{~B}-\mathrm{H} 44$ & 0.9700 \\
$\mathrm{O} 6-\mathrm{H} 6 \mathrm{D}$ & $0.84(6)$ & $\mathrm{C} 5-\mathrm{H} 51$ & 0.9700 \\
$\mathrm{O} 6-\mathrm{H} 6 \mathrm{C}$ & $0.86(5)$ & $\mathrm{C} 5-\mathrm{H} 54$ & 0.9700 \\
$\mathrm{~N} 1-\mathrm{C} 3$ & $0.85(7)$ & $\mathrm{C} 5-\mathrm{H} 52$ & 0.9700 \\
$\mathrm{~N} 1-\mathrm{C} 2$ & $0.83(6)$ & $\mathrm{C} 5-\mathrm{H} 53$ & 0.9700
\end{tabular}




\begin{tabular}{|c|c|}
\hline $\mathrm{O} 1-\mathrm{Sr} 1-\mathrm{O} 3$ & $137.44(18)$ \\
\hline $\mathrm{O} 1-\mathrm{Sr} 1-\mathrm{O} 4$ & $138.19(17)$ \\
\hline $\mathrm{O} 1-\mathrm{Sr} 1-\mathrm{O} 5$ & 70.37 (18) \\
\hline $\mathrm{O} 1-\mathrm{Sr} 1-\mathrm{O} 6$ & $73.32(18)$ \\
\hline $\mathrm{O} 1-\mathrm{Sr} 1-\mathrm{O} 2^{\mathrm{i}}$ & $129.10(14)$ \\
\hline $\mathrm{O} 1-\mathrm{Sr} 1-\mathrm{O}^{3 i}$ & $69.11(17)$ \\
\hline $\mathrm{O} 1-\mathrm{Sr} 1-\mathrm{O} 4^{\mathrm{ii}}$ & $70.35(17)$ \\
\hline $\mathrm{O} 1-\mathrm{Sr} 1-\mathrm{O} 2^{\mathrm{iii}}$ & $112.66(13)$ \\
\hline $\mathrm{O} 3-\mathrm{Sr} 1-\mathrm{O} 4$ & $84.36(18)$ \\
\hline $\mathrm{O} 3-\mathrm{Sr} 1-\mathrm{O} 5$ & $145.76(18)$ \\
\hline $\mathrm{O} 3-\mathrm{Sr} 1-\mathrm{O} 6$ & $71.85(19)$ \\
\hline $\mathrm{O} 2^{\mathrm{i}}-\mathrm{Sr} 1-\mathrm{O} 3$ & $62.82(16)$ \\
\hline $\mathrm{O} 3-\mathrm{Sr} 1-\mathrm{O}^{3 \mathrm{ii}}$ & 133.75 (19) \\
\hline $\mathrm{O} 3-\mathrm{Sr} 1-\mathrm{O} 4^{\mathrm{ii}}$ & $77.67(17)$ \\
\hline $\mathrm{O} 22^{\mathrm{iii}}-\mathrm{Sr} 1-\mathrm{O} 3$ & $72.95(16)$ \\
\hline $\mathrm{O} 4-\mathrm{Sr} 1-\mathrm{O} 5$ & $71.86(18)$ \\
\hline $\mathrm{O} 4-\mathrm{Sr} 1-\mathrm{O} 6$ & $137.87(18)$ \\
\hline $\mathrm{O} 22^{\mathrm{i}}-\mathrm{Sr} 1-\mathrm{O} 4$ & $62.60(16)$ \\
\hline $\mathrm{O} 3^{\mathrm{ii}}-\mathrm{Sr} 1-\mathrm{O} 4$ & $80.08(17)$ \\
\hline $\mathrm{O} 4-\mathrm{Sr} 1-\mathrm{O}^{\mathrm{ii}}$ & 133.67 (19) \\
\hline $\mathrm{O} 22^{\mathrm{iii}}-\mathrm{Sr} 1-\mathrm{O} 4$ & $72.62(16)$ \\
\hline $\mathrm{O} 5-\mathrm{Sr} 1-\mathrm{O} 6$ & $110.10(19)$ \\
\hline $\mathrm{O} 22^{\mathrm{i}}-\mathrm{Sr} 1-\mathrm{O} 5$ & $84.14(17)$ \\
\hline $\mathrm{O} 3^{\mathrm{ii}}-\mathrm{Sr} 1-\mathrm{O} 5$ & $66.80(19)$ \\
\hline $\mathrm{O} 44^{\mathrm{ii}}-\mathrm{Sr} 1-\mathrm{O} 5$ & $136.53(18)$ \\
\hline $\mathrm{O} 2^{\mathrm{iii}}-\mathrm{Sr} 1-\mathrm{O} 5$ & $120.21(17)$ \\
\hline $\mathrm{O} 22^{\mathrm{i}}-\mathrm{Sr} 1-\mathrm{O} 6$ & $75.56(17)$ \\
\hline $\mathrm{O}^{\mathrm{ii}}-\mathrm{Sr} 1-\mathrm{O} 6$ & $140.90(18)$ \\
\hline $\mathrm{O} 4 \mathrm{ii}-\mathrm{Sr} 1-\mathrm{O} 6$ & $75.16(19)$ \\
\hline $\mathrm{O}^{2 \mathrm{ii}}-\mathrm{Sr} 1-\mathrm{O} 6$ & $128.45(18)$ \\
\hline $\mathrm{O} 2^{\mathrm{i}}-\mathrm{Sr} 1-\mathrm{O}^{\mathrm{ii}}$ & $138.60(17)$ \\
\hline $\mathrm{O} 2^{\mathrm{i}}-\mathrm{Sr} 1-\mathrm{O} 4^{\mathrm{ii}}$ & $136.37(16)$ \\
\hline $\mathrm{O} 2^{\mathrm{i}}-\mathrm{Sr} 1-\mathrm{O} 2^{\mathrm{iii}}$ & $118.24(13)$ \\
\hline $\mathrm{O}^{\mathrm{ii}}-\mathrm{Sr} 1-\mathrm{O} 4^{\mathrm{ii}}$ & $82.36(17)$ \\
\hline  & $60.87(16)$ \\
\hline $\mathrm{O} 2^{\mathrm{iii}}-\mathrm{Sr} 1-\mathrm{O} 4^{\mathrm{ii}}$ & $61.37(16)$ \\
\hline $\mathrm{Sr} 1-\mathrm{O} 1-\mathrm{C} 1$ & $144.8(4)$ \\
\hline $\mathrm{Sr} 1^{\mathrm{iv}}-\mathrm{O} 2-\mathrm{C} 1$ & $144.7(4)$ \\
\hline $\mathrm{Sr} 1^{\mathrm{ii}}-\mathrm{O} 2-\mathrm{C} 1$ & $124.2(4)$ \\
\hline $\mathrm{Sr} 1^{\mathrm{iv}}-\mathrm{O} 2-\mathrm{Sr} 1^{\mathrm{ii}}$ & $90.87(13)$ \\
\hline $\mathrm{Sr} 1-\mathrm{O} 3-\mathrm{Sr} 1^{i \mathrm{ii}}$ & $95.2(2)$ \\
\hline $\mathrm{Sr} 1-\mathrm{O} 4-\mathrm{Sr} 1^{i \mathrm{ii}}$ & $96.47(17)$ \\
\hline $\mathrm{H} 3 \mathrm{C}-\mathrm{O} 3-\mathrm{H} 3 \mathrm{D}$ & $111(6)$ \\
\hline 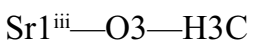 & $115(5)$ \\
\hline $\mathrm{Sr}^{\mathrm{iii}}-\mathrm{O} 3-\mathrm{H} 3 \mathrm{D}$ & $110(5)$ \\
\hline $\mathrm{Sr} 1-\mathrm{O} 3-\mathrm{H} 3 \mathrm{C}$ & $119(6)$ \\
\hline $\mathrm{Sr} 1-\mathrm{O} 3-\mathrm{H} 3 \mathrm{D}$ & $107(5)$ \\
\hline $\mathrm{H} 4 \mathrm{E}-\mathrm{O} 4-\mathrm{H} 4 \mathrm{~F}$ & $111(6)$ \\
\hline
\end{tabular}

\begin{tabular}{|c|c|}
\hline $\mathrm{Sr} 1-\mathrm{O} 6-\mathrm{H} 6 \mathrm{D}$ & $130(6)$ \\
\hline $\mathrm{Sr} 1-\mathrm{O} 6-\mathrm{H} 6 \mathrm{C}$ & $119(5)$ \\
\hline $\mathrm{H} 6 \mathrm{C}-\mathrm{O} 6-\mathrm{H} 6 \mathrm{D}$ & $111(8)$ \\
\hline $\mathrm{C} 2-\mathrm{N} 1-\mathrm{C} 3$ & $107.2(5)$ \\
\hline $\mathrm{C} 3-\mathrm{N} 1-\mathrm{H} 1 \mathrm{~B}$ & $117(6)$ \\
\hline $\mathrm{H} 1 \mathrm{~A}-\mathrm{N} 1-\mathrm{H} 1 \mathrm{~B}$ & $97(5)$ \\
\hline $\mathrm{C} 2-\mathrm{N} 1-\mathrm{H} 1 \mathrm{~A}$ & $114(5)$ \\
\hline $\mathrm{C} 2-\mathrm{N} 1-\mathrm{H} 1 \mathrm{~B}$ & $115(5)$ \\
\hline $\mathrm{C} 3-\mathrm{N} 1-\mathrm{H} 1 \mathrm{~A}$ & $105(5)$ \\
\hline $\mathrm{O} 1-\mathrm{C} 1-\mathrm{C} 2$ & $115.4(5)$ \\
\hline $\mathrm{O} 2-\mathrm{C} 1-\mathrm{C} 2$ & $117.0(5)$ \\
\hline $\mathrm{O} 1-\mathrm{C} 1-\mathrm{O} 2$ & $127.6(6)$ \\
\hline $\mathrm{N} 1-\mathrm{C} 2-\mathrm{C} 5$ & $102.2(6)$ \\
\hline $\mathrm{N} 1-\mathrm{C} 2-\mathrm{C} 1$ & $112.2(5)$ \\
\hline $\mathrm{C} 1-\mathrm{C} 2-\mathrm{C} 5$ & $117.6(6)$ \\
\hline $\mathrm{N} 1-\mathrm{C} 3-\mathrm{C} 4 \mathrm{~B}$ & $104.6(10)$ \\
\hline $\mathrm{N} 1-\mathrm{C} 3-\mathrm{C} 4 \mathrm{~A}$ & $105.7(8)$ \\
\hline $\mathrm{C} 3-\mathrm{C} 4 \mathrm{~A}-\mathrm{C} 5$ & $104.7(10)$ \\
\hline $\mathrm{C} 3-\mathrm{C} 4 \mathrm{~B}-\mathrm{C} 5$ & $104.8(19)$ \\
\hline $\mathrm{C} 2-\mathrm{C} 5-\mathrm{C} 4 \mathrm{~A}$ & $101.1(12)$ \\
\hline $\mathrm{C} 2-\mathrm{C} 5-\mathrm{C} 4 \mathrm{~B}$ & $108.8(15)$ \\
\hline $\mathrm{N} 1-\mathrm{C} 2-\mathrm{H} 2$ & 108.00 \\
\hline $\mathrm{C} 1-\mathrm{C} 2-\mathrm{H} 2$ & 108.00 \\
\hline $\mathrm{C} 5-\mathrm{C} 2-\mathrm{H} 2$ & 108.00 \\
\hline $\mathrm{N} 1-\mathrm{C} 3-\mathrm{H} 31$ & 111.00 \\
\hline $\mathrm{N} 1-\mathrm{C} 3-\mathrm{H} 32$ & 111.00 \\
\hline $\mathrm{N} 1-\mathrm{C} 3-\mathrm{H} 33$ & 111.00 \\
\hline $\mathrm{N} 1-\mathrm{C} 3-\mathrm{H} 34$ & 111.00 \\
\hline $\mathrm{C} 4 \mathrm{~A}-\mathrm{C} 3-\mathrm{H} 31$ & 111.00 \\
\hline $\mathrm{C} 4 \mathrm{~A}-\mathrm{C} 3-\mathrm{H} 32$ & 111.00 \\
\hline $\mathrm{H} 31-\mathrm{C} 3-\mathrm{H} 32$ & 109.00 \\
\hline $\mathrm{C} 4 \mathrm{~B}-\mathrm{C} 3-\mathrm{H} 33$ & 111.00 \\
\hline $\mathrm{C} 4 \mathrm{~B}-\mathrm{C} 3-\mathrm{H} 34$ & 111.00 \\
\hline $\mathrm{H} 33-\mathrm{C} 3-\mathrm{H} 34$ & 109.00 \\
\hline $\mathrm{C} 3-\mathrm{C} 4 \mathrm{~A}-\mathrm{H} 41$ & 111.00 \\
\hline $\mathrm{C} 3-\mathrm{C} 4 \mathrm{~A}-\mathrm{H} 42$ & 111.00 \\
\hline $\mathrm{C} 5-\mathrm{C} 4 \mathrm{~A}-\mathrm{H} 41$ & 111.00 \\
\hline $\mathrm{C} 5-\mathrm{C} 4 \mathrm{~A}-\mathrm{H} 42$ & 111.00 \\
\hline $\mathrm{H} 41-\mathrm{C} 4 \mathrm{~A}-\mathrm{H} 42$ & 109.00 \\
\hline $\mathrm{H} 43-\mathrm{C} 4 \mathrm{~B}-\mathrm{H} 44$ & 109.00 \\
\hline $\mathrm{C} 3-\mathrm{C} 4 \mathrm{~B}-\mathrm{H} 44$ & 111.00 \\
\hline $\mathrm{C} 5-\mathrm{C} 4 \mathrm{~B}-\mathrm{H} 43$ & 111.00 \\
\hline $\mathrm{C} 3-\mathrm{C} 4 \mathrm{~B}-\mathrm{H} 43$ & 111.00 \\
\hline $\mathrm{C} 5-\mathrm{C} 4 \mathrm{~B}-\mathrm{H} 44$ & 111.00 \\
\hline $\mathrm{C} 2-\mathrm{C} 5-\mathrm{H} 52$ & 112.00 \\
\hline $\mathrm{C} 2-\mathrm{C} 5-\mathrm{H} 53$ & 110.00 \\
\hline $\mathrm{C} 2-\mathrm{C} 5-\mathrm{H} 51$ & 112.00 \\
\hline $\mathrm{C} 4 \mathrm{~B}-\mathrm{C} 5-\mathrm{H} 54$ & 110.00 \\
\hline
\end{tabular}




\begin{tabular}{|c|c|c|c|}
\hline $\mathrm{Sr} 1-\mathrm{O} 4-\mathrm{H} 4 \mathrm{E}$ & $117(5)$ & $\mathrm{H} 53-\mathrm{C} 5-\mathrm{H} 54$ & 108.00 \\
\hline $\mathrm{Sr} 1-\mathrm{O} 4-\mathrm{H} 4 \mathrm{~F}$ & $111(4)$ & $\mathrm{C} 2-\mathrm{C} 5-\mathrm{H} 54$ & 110.00 \\
\hline 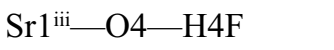 & $107(4)$ & $\mathrm{C} 4 \mathrm{~A}-\mathrm{C} 5-\mathrm{H} 51$ & 112.00 \\
\hline 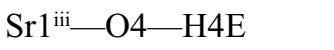 & $112(5)$ & $\mathrm{C} 4 \mathrm{~A}-\mathrm{C} 5-\mathrm{H} 52$ & 112.00 \\
\hline $\mathrm{Sr} 1-\mathrm{O} 5-\mathrm{H} 5 \mathrm{D}$ & $119(5)$ & $\mathrm{H} 51-\mathrm{C} 5-\mathrm{H} 52$ & 109.00 \\
\hline $\mathrm{Sr} 1-\mathrm{O} 5-\mathrm{H} 5 \mathrm{C}$ & $118(5)$ & $\mathrm{C} 4 \mathrm{~B}-\mathrm{C} 5-\mathrm{H} 53$ & 110.00 \\
\hline $\mathrm{H} 5 \mathrm{C}-\mathrm{O} 5-\mathrm{H} 5 \mathrm{D}$ & $109(7)$ & & \\
\hline $\mathrm{O} 3-\mathrm{Sr} 1-\mathrm{O} 1-\mathrm{C} 1$ & $-76.6(8)$ & $\mathrm{O} 5^{\mathrm{iii}}-\mathrm{Sr} 1^{\mathrm{iii}}-\mathrm{O} 3-\mathrm{Sr} 1$ & $-158.1(2)$ \\
\hline $\mathrm{O} 4-\mathrm{Sr} 1-\mathrm{O} 1-\mathrm{C} 1$ & $101.4(8)$ & $\mathrm{O}^{\mathrm{iii}}-\mathrm{Sr} 1^{\mathrm{iii}}-\mathrm{O} 3-\mathrm{Sr} 1$ & $-64.4(3)$ \\
\hline $\mathrm{O} 5-\mathrm{Sr} 1-\mathrm{O} 1-\mathrm{C} 1$ & $128.0(8)$ & $\mathrm{O} 1-\mathrm{Sr} 1-\mathrm{O} 4-\mathrm{Sr}^{\mathrm{iii}}$ & $171.61(16)$ \\
\hline $\mathrm{O} 6-\mathrm{Sr} 1-\mathrm{O} 1-\mathrm{C} 1$ & $-112.8(8)$ & $\mathrm{O} 3-\mathrm{Sr} 1-\mathrm{O} 4-\mathrm{Sr} 1^{\mathrm{iii}}$ & $-9.71(18)$ \\
\hline $\mathrm{O} 2-\mathrm{Sr} 1-\mathrm{O} 1-\mathrm{C} 1$ & $-167.5(7)$ & $\mathrm{O} 5-\mathrm{Sr} 1-\mathrm{O} 4-\mathrm{Sr} 1^{\mathrm{iii}}$ & $145.3(2)$ \\
\hline $\mathrm{O}^{\mathrm{ii}}-\mathrm{Sr} 1-\mathrm{O} 1-\mathrm{C} 1$ & $56.1(8)$ & $\mathrm{O} 6-\mathrm{Sr} 1-\mathrm{O} 4-\mathrm{Sr} 1^{\mathrm{iii}}$ & $45.1(3)$ \\
\hline $\mathrm{O} 4^{\mathrm{ii}}-\mathrm{Sr} 1-\mathrm{O} 1-\mathrm{C} 1$ & $-33.0(8)$ & $\mathrm{O} 2^{\mathrm{i}}-\mathrm{Sr} 1-\mathrm{O} 4-\mathrm{Sr} 1^{\mathrm{iii}}$ & $52.53(16)$ \\
\hline $\mathrm{O} 2^{\mathrm{iii}-} \mathrm{Sr} 1-\mathrm{O} 1-\mathrm{C} 1$ & $12.5(8)$ & $\mathrm{O} 3^{\mathrm{ii}}-\mathrm{Sr} 1-\mathrm{O} 4-\mathrm{Sr} 1^{\mathrm{iii}}$ & $-146.0(2)$ \\
\hline $\mathrm{O} 1^{\mathrm{iv}}-\mathrm{Sr} 1^{\mathrm{iv}}-\mathrm{O} 2-\mathrm{C} 1$ & $5.3(8)$ & $\mathrm{O} 4^{\mathrm{ii}}-\mathrm{Sr} 1-\mathrm{O} 4-\mathrm{Sr} 1^{\mathrm{iii}}$ & $-76.7(3)$ \\
\hline $\mathrm{O}^{\text {iv }}-\mathrm{Sr} 1^{\text {iv }}-\mathrm{O} 2-\mathrm{C} 1$ & $-125.2(8)$ & $\mathrm{O} 2^{\mathrm{iii}}-\mathrm{Sr} 1-\mathrm{O} 4-\mathrm{Sr} 1^{\mathrm{iii}}$ & $-83.58(18)$ \\
\hline $\mathrm{O} 4^{\mathrm{iv}}-\mathrm{Sr} 1^{\mathrm{iv}}-\mathrm{O} 2-\mathrm{C} 1$ & $136.7(8)$ & $\mathrm{O} 3-\mathrm{Sr} 1^{\mathrm{iii}}-\mathrm{O} 4-\mathrm{Sr} 1$ & $9.45(18)$ \\
\hline $\mathrm{O} 5^{\mathrm{iv}}-\mathrm{Sr} 1^{\mathrm{iv}}-\mathrm{O} 2-\mathrm{C} 1$ & $64.1(8)$ & $\mathrm{O} 2^{\mathrm{i}}-\mathrm{Sr} 1^{\mathrm{iii}}-\mathrm{O} 4-\mathrm{Sr} 1$ & $-51.45(16)$ \\
\hline $\mathrm{O}^{\mathrm{iv}}-\mathrm{Sr} 1^{\mathrm{iv}}-\mathrm{O} 2-\mathrm{C} 1$ & $-48.4(8)$ & 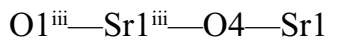 & $79.93(18)$ \\
\hline $\mathrm{O} 2^{\mathrm{ii}}-\mathrm{Sr} 1^{\mathrm{iv}}-\mathrm{O} 2-\mathrm{C} 1$ & $-174.6(7)$ & 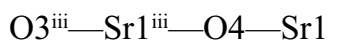 & $-128.6(2)$ \\
\hline $\mathrm{O}^{\mathrm{iv}}-\mathrm{Sr} 1^{1 \mathrm{ii}}-\mathrm{O} 2-\mathrm{C} 1$ & $127.5(6)$ & $\mathrm{O} 4^{\mathrm{iii}}-\mathrm{Sr} 1^{\mathrm{iii}}-\mathrm{O} 4-\mathrm{Sr} 1$ & $-58.9(3)$ \\
\hline $\mathrm{O} 4^{\mathrm{iv}}-\mathrm{Sr} 1^{1 \mathrm{ii}}-\mathrm{O} 2-\mathrm{C} 1$ & $-135.0(6)$ & $\mathrm{O}^{\mathrm{iii}}-\mathrm{Sr} 1^{\mathrm{iii}}-\mathrm{O} 4-\mathrm{Sr} 1$ & $53.5(3)$ \\
\hline $\mathrm{O} 1^{\mathrm{ii}}-\mathrm{Sr} 1^{\mathrm{ii}}-\mathrm{O} 2-\mathrm{C} 1$ & $175.1(5)$ & $\mathrm{O}^{\mathrm{iii}}-\mathrm{Sr} 1^{\mathrm{iii}}-\mathrm{O} 4-\mathrm{Sr} 1$ & $157.2(2)$ \\
\hline $\mathrm{O}^{3 \mathrm{ii}}-\mathrm{Sr} 1^{\mathrm{ii}}-\mathrm{O} 2-\mathrm{C} 1$ & $-50.0(5)$ & $\mathrm{Sr} 1-\mathrm{O} 1-\mathrm{C} 1-\mathrm{O} 2$ & $-19.8(13)$ \\
\hline $\mathrm{O} 4^{\mathrm{ii}}-\mathrm{Sr} 1^{\mathrm{ii}}-\mathrm{O} 2-\mathrm{C} 1$ & $39.4(5)$ & $\mathrm{Sr} 1-\mathrm{O} 1-\mathrm{C} 1-\mathrm{C} 2$ & $158.9(6)$ \\
\hline $\mathrm{O} 5^{\mathrm{ii}}-\mathrm{Sr} 1^{\mathrm{ii}}-\mathrm{O} 2-\mathrm{C} 1$ & $95.3(5)$ & $\mathrm{Sr} 1^{\mathrm{iv}}-\mathrm{O} 2-\mathrm{C} 1-\mathrm{O} 1$ & $-172.8(5)$ \\
\hline $\mathrm{O}^{\mathrm{ii}}-\mathrm{Sr} 1^{\mathrm{ii}}-\mathrm{O} 2-\mathrm{C} 1$ & $-98.7(5)$ & $\mathrm{Sr} 1^{\mathrm{ii}}-\mathrm{O} 2-\mathrm{C} 1-\mathrm{O} 1$ & $13.2(10)$ \\
\hline $\mathrm{O} 2^{\mathrm{iii}-}-\mathrm{Sr} 1^{\mathrm{ii}}-\mathrm{O} 2-\mathrm{C} 1$ & $-5.0(6)$ & $\mathrm{Sr} 1^{\mathrm{iv}}-\mathrm{O} 2-\mathrm{C} 1-\mathrm{C} 2$ & $8.5(11)$ \\
\hline $\mathrm{O} 1-\mathrm{Sr} 1-\mathrm{O} 3-\mathrm{Sr} 1^{\mathrm{iii}}$ & $-171.81(15)$ & $\mathrm{Sr} 1^{\mathrm{ii}}-\mathrm{O} 2-\mathrm{C} 1-\mathrm{C} 2$ & $-165.5(4)$ \\
\hline $\mathrm{O} 4-\mathrm{Sr} 1-\mathrm{O} 3-\mathrm{Sr} 1^{\mathrm{iii}}$ & $9.48(18)$ & $\mathrm{C} 2-\mathrm{N} 1-\mathrm{C} 3-\mathrm{C} 4 \mathrm{~A}$ & $-10.6(17)$ \\
\hline $\mathrm{O} 5-\mathrm{Sr} 1-\mathrm{O} 3-\mathrm{Sr} 1^{\mathrm{iii}}$ & $-36.0(4)$ & $\mathrm{C} 3-\mathrm{N} 1-\mathrm{C} 2-\mathrm{C} 5$ & $34.0(8)$ \\
\hline $\mathrm{O} 6-\mathrm{Sr} 1-\mathrm{O} 3-\mathrm{Sr} 1^{\mathrm{iii}}$ & $-135.3(2)$ & $\mathrm{C} 3-\mathrm{N} 1-\mathrm{C} 2-\mathrm{C} 1$ & $160.9(6)$ \\
\hline $\mathrm{O} 2^{\mathrm{i}}-\mathrm{Sr} 1-\mathrm{O} 3-\mathrm{Sr} 1^{\mathrm{iii}}$ & $-52.54(16)$ & $\mathrm{O} 1-\mathrm{C} 1-\mathrm{C} 2-\mathrm{N} 1$ & $4.6(9)$ \\
\hline $\mathrm{O}{ }^{\mathrm{ii}}-\mathrm{Sr} 1-\mathrm{O} 3-\mathrm{Sr} 1^{\mathrm{iii}}$ & $80.0(3)$ & $\mathrm{O} 2-\mathrm{C} 1-\mathrm{C} 2-\mathrm{C} 5$ & $-58.5(9)$ \\
\hline $\mathrm{O} 4^{\mathrm{ii}}-\mathrm{Sr} 1-\mathrm{O} 3-\mathrm{Sr} 1^{\mathrm{iii}}$ & $146.5(2)$ & $\mathrm{O} 2-\mathrm{C} 1-\mathrm{C} 2-\mathrm{N} 1$ & $-176.6(6)$ \\
\hline 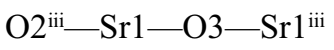 & 83.01 (17) & $\mathrm{O} 1-\mathrm{C} 1-\mathrm{C} 2-\mathrm{C} 5$ & $122.7(7)$ \\
\hline $\mathrm{O} 4-\mathrm{Sr} 1^{\mathrm{iii}-\mathrm{O} 3-\mathrm{Sr} 1}$ & $-9.45(18)$ & $\mathrm{N} 1-\mathrm{C} 2-\mathrm{C} 5-\mathrm{C} 4 \mathrm{~A}$ & $-43.4(12)$ \\
\hline  & $51.95(16)$ & $\mathrm{C} 1-\mathrm{C} 2-\mathrm{C} 5-\mathrm{C} 4 \mathrm{~A}$ & $-166.7(12)$ \\
\hline $\mathrm{O} 1^{\mathrm{iii}-\mathrm{Sr}} 1^{\mathrm{iii}-\mathrm{O} 3-\mathrm{Sr} 1}$ & $-81.27(18)$ & $\mathrm{N} 1-\mathrm{C} 3-\mathrm{C} 4 \mathrm{~A}-\mathrm{C} 5$ & $-17(2)$ \\
\hline $\mathrm{O}{ }^{\mathrm{iii}}-\mathrm{Sr} 1^{\mathrm{iii}-\mathrm{O} 3}-\mathrm{Sr} 1$ & $55.3(3)$ & $\mathrm{C} 3-\mathrm{C} 4 \mathrm{~A}-\mathrm{C} 5-\mathrm{C} 2$ & $37(2)$ \\
\hline $\mathrm{O} 4^{\mathrm{iii}-\mathrm{Sr}} 1^{\mathrm{iii}-\mathrm{O} 3-\mathrm{Sr} 1}$ & $127.5(2)$ & & \\
\hline
\end{tabular}

Symmetry codes: (i) $x+1, y, z$; (ii) $x-1 / 2,-y+1 / 2,-z+1$; (iii) $x+1 / 2,-y+1 / 2,-z+1$; (iv) $x-1, y, z$. 


\section{supporting information}

Hydrogen-bond geometry $\left(\AA,{ }^{\circ}\right)$

\begin{tabular}{lllll}
\hline$D-\mathrm{H} \cdots A$ & $D-\mathrm{H}$ & $\mathrm{H} \cdots A$ & $D \cdots A$ & $D-\mathrm{H} \cdots A$ \\
\hline $\mathrm{N} 1-\mathrm{H} 1 A^{\cdots} \cdots \mathrm{Br} 2^{\mathrm{i}}$ & $0.90(6)$ & $2.52(5)$ & $3.374(7)$ & $159(6)$ \\
$\mathrm{N} 1-\mathrm{H} 1 B \cdots \mathrm{Br} 3^{\mathrm{i}}$ & $0.90(7)$ & $2.40(7)$ & $3.240(7)$ & $156(8)$ \\
$\mathrm{O} 3-\mathrm{H} 3 C \cdots \mathrm{Br} 3^{\mathrm{v}}$ & $0.84(7)$ & $2.63(7)$ & $3.440(6)$ & $163(7)$ \\
$\mathrm{O} 3-\mathrm{H} 3 D^{\cdots} \cdots \mathrm{Br}^{\mathrm{vi}}$ & $0.84(7)$ & $2.54(7)$ & $3.376(6)$ & $172(5)$ \\
$\mathrm{O} 4-\mathrm{H} 4 E \cdots \mathrm{Br} 2^{\mathrm{vii}}$ & $0.85(6)$ & $2.47(7)$ & $3.281(6)$ & $162(7)$ \\
$\mathrm{O} 4-\mathrm{H} 4 F \cdots \mathrm{Br} 3^{\text {viii }}$ & $0.83(6)$ & $2.52(6)$ & $3.347(6)$ & $174(6)$ \\
$\mathrm{O} 5-\mathrm{H} 5 C \cdots \mathrm{Br} 2^{\mathrm{i}}$ & $0.86(5)$ & $2.54(5)$ & $3.369(6)$ & $164(6)$ \\
$\mathrm{O} 5-\mathrm{H} 5 D \cdots \mathrm{Br} 3^{\text {viii }}$ & $0.84(6)$ & $2.48(6)$ & $3.304(6)$ & $166(6)$ \\
$\mathrm{O} 6-\mathrm{H} 6 C \cdots \mathrm{Br} 2^{\mathrm{vi}}$ & $0.83(6)$ & $2.58(6)$ & $3.393(6)$ & $167(5)$ \\
$\mathrm{O} 6-\mathrm{H} 6 D \cdots \mathrm{Br} 3^{\mathrm{i}}$ & $0.85(7)$ & $2.56(6)$ & $3.378(6)$ & $162(7)$
\end{tabular}

Symmetry codes: (i) $x+1, y, z$; (v) $-x+3 / 2,-y+1, z+1 / 2$; (vi) $-x+2, y+1 / 2,-z+1 / 2$; (vii) $-x+3 / 2,-y, z+1 / 2$; (viii) $-x+2, y-1 / 2,-z+1 / 2$. 